\title{
Can contrast-enhanced mammography replace dynamic contrast-enhanced MRI in the assessment of sonomammographic indeterminate breast lesions?
}

Rasha Mohamed Kamal ${ }^{1}$, Mennatallah Mohamed Hanafy ${ }^{1 *}$, Sahar Mahmoud Mansour ${ }^{1}$, Maher Hassan² and Mohamed Mohamed Gomaa ${ }^{3}$

\begin{abstract}
Background: Dynamic contrast-enhanced MRI of the breast has been used for several years in the assessment of indeterminate mammographic findings. Contrast-enhanced mammography is a relatively novel imaging technique that has shown comparable sensitivity and specificity to MRI. Contrast-enhanced mammography is a relatively easy feasible study with high sensitivity and low cost. Our aim was to assess the feasibility of replacing dynamic contrastenhanced (DCE)-MRI by contrast-enhanced mammography in the assessment of sonomammographic indeterminate lesions (BIRADS 3 and 4).

Results: The study included 82 patients with 171 breast lesions. They all performed contrast-enhanced mammography and dynamic contrast-enhanced MRI. DCE-MRI sensitivity and NPV were significantly higher than those of contrast-enhanced mammogram (CEM). The overall accuracy of MRI was better than that of CEM; however, no statistically significant difference could be detected.

Conclusion: Contrast-enhanced mammography and dynamic contrast-enhanced MRI improved the characterization of breast lesions. CEM showed slightly lower sensitivity and accuracy compared to MRl; however, because of being relatively easy, available, cheap, and acceptable by women, CEM can replace DC-MRI as a problem-solving tool in the characterization of indeterminate breast lesions.
\end{abstract}

Keywords: Contrast-enhanced mammogram, Dynamic contrast-enhanced MRI, indeterminate breast lesions

\section{Background}

Breast cancer is a major health problem and a leading cause of death among women in Egypt. Early detection of breast cancer aims to reduce morbidity and mortality rates. Mammography has been established as the imaging modality for screening and early detection of breast cancer; however, it is accused of having low sensitivity and specificity in women with dense breasts [1].

\footnotetext{
* Correspondence: drmennamhanafy@gmail.com

'Department of Diagnostic and Interventional Radiology, Faculty of Medicine, Cairo University, Cairo, Egypt

Full list of author information is available at the end of the article
}

Accurate diagnosis and characterization of breast lesions have an essential role in the management and improved prognosis of breast cancer $[2,3]$.

CE-MRI is used nowadays as a problem-solving tool and as an adjunct to sonomammography in women at high risk or those with extremely dense breasts [4].

Dynamic contrast-enhanced (DCE)-MRI allows better characterization of lesions through morphologic and kinematic assessment after administration of contrast material [5].

Lesion characterization by DCE-MRI depends on the difference in vascular supply between normal and 
neoplastic tissue. Neoplastic tissue demonstrates contrast uptake due to the development of neovascularization [6].

However, MRI is a relatively expensive study that necessitates at least 30 to $40 \mathrm{~min}$ to acquire images and is not equally accessible to all women [2].

DCE-MRI is accused of having low specificity and being not recommended in the assessment of microcalcifications [7].

Contrast-enhanced mammography (CEM) is one of the relatively novel imaging modalities. CEM is a relatively easy feasible study with high sensitivity and low cost [2]. It provides low-energy images comparable to mammographic images and post-contrast recombined images to evaluate tumor neovascularity $[8,9]$.

CEM allows better evaluation of calcifications by their visualization on low-energy images combined with enhancement criteria on the contrast-enhanced recombined images [10]. Dual contrast-enhanced mammography is the commonly used technique; this technique lacks kinematic information [8].

Our aim was to assess the feasibility of replacing CE MRI by contrast-enhanced spectral mammography in the assessment of sonomammographic indeterminate breast lesions (BIRADS 3 and 4) in contrast to most of the previous studies which were concerned upon sonomammographic suspicious breast lesions (BIRADS 4 and 5)

\section{Methods}

This study included 171 lesions in 82 patients, 20 of them had bilateral breast lesions. Their ages ranged from 29 to 71 years (mean age $49.298 \pm 10.75$ ). The study was approved by the ethical committee and informed written consent was taken from all subjects.

\section{Subjects \\ Inclusion criteria}

1. Patients with at least a single indeterminate lesion (BIRADS 3 and 4)

\section{Exclusion criteria}

1. Patients with a negative mammogram (BIRADS 1) or those with definite benign criteria (BIRADS 2)

2. Contraindication to mammography, e.g., Pregnant women

3. Contraindication to IV contrast, e.g., patients with renal impairment, allergic patients, or those known to have a history of anaphylactic reaction from contrast media

4. Contraindication to MRI, e.g., cardiac pacemaker, aneurysmal clips, and bone growth stimulators

\section{Contrast-enhanced mammography technique}

CEM examination was performed using Senographe Essential, GE healthcare full-field digital mammography machine, with sonobright.

A one-shot intravenous injection (of $1.5 \mathrm{~mL} / \mathrm{kg}$ ) of non-ionic contrast media was performed. Two minutes after contrast administration, a low-energy (23-32 KVp) and high-energy $(45-49 \mathrm{KVp})$ pair of images were acquired within $20 \mathrm{~s}$ of one another in mediolateral oblique (MLO) view and then in craniocaudal (CC) position. Recombined iodine-enhanced images were obtained by the subtraction of low- and high-energy images.

\section{Dynamic contrast-enhanced MRI technique}

MRI was performed using a Siemens 1.5-T MRI system.

The examination was performed using a bilateral breast surface coil with the patient in the prone position.

The imaging studies included localizer in the sagittal or coronal orientations; pre-contrast series: axial T1weighted turbo spin-echo (TR/TE $=307 / 4.6 \mathrm{~ms}$ ), axial T2-weighted turbo spin-echo (TR/TE $=4.3 \mathrm{~s} / 91 \mathrm{~ms})$, and axial short tau inversion recovery (STIR) $(\mathrm{TR} / \mathrm{TE}=$ $5.2 \mathrm{~s} / 71 \mathrm{~ms}$; inversion time $=170 \mathrm{~ms}$ ) which was a fat suppression technique with inversion time as well as sagittal T2 turbo spin-echo weighted sequences; and postcontrast series: seven dynamic acquisitions, one before and sex after intravenous injection $0.1 \mathrm{mmol} / \mathrm{kg}$ bodyweight of contrast material (Gd-DTPA), using the dynamic THRIVE sequence (T1 high-resolution isotropic volumetric examination) $(\mathrm{TR} / \mathrm{TE}=5 / 2 \mathrm{~ms})$.

The time interval between the two techniques ranged from 1 week to month duration. The kidney function was assessed before each modality.

\section{Image analysis and interpretation of contrast-enhanced mammography and contrast-enhanced MRI}

The 2013 MRI BIRADS lexicon was used in the characterization of detected lesions in both CEM and DCE-MRI with BIRADS category given to each lesion. CEM and CE-MRI were assessed for the presence or absence of enhancing lesions. Enhancing lesions were then classified as mass or non-mass. When an enhancing mass lesion was detected, it was further assessed for its margins (circumscribed, not circumscribed irregular, or not circumscribed speculated), degree of enhancement (mild, moderate, and severe), and pattern of internal enhancement (homogenous, heterogeneous, septations, or ring enhancement). When enhancing non-mass lesion was detected, it was further assessed for distribution (focal, linear, segmental, regional, multiregional, or diffuse), pattern of internal enhancement (homogenous, heterogeneous, clustered, and clumped), and degree of enhancement (mild, moderate, and severe). 
Low-energy images of CEM were assessed in reference to the mammography 2013 BIRADS lexicon. Nonenhancing lesions on DCE-MRI were assessed in T1, T2, and STIR images.

\section{Statistical analysis}

Data were coded and entered using the statistical package SPSS (Statistical Package for the Social Sciences) version 25. Data were summarized using mean, standard deviation, median, minimum, and maximum in quantitative data and using frequency (count) and relative frequency (percentage) for categorical data. For comparing categorical data, chi-square $\left(\chi^{2}\right)$ test was performed. Correlations between quantitative variables were done using Spearman correlation coefficient. Standard diagnostic indices including sensitivity, specificity, positive predictive value (PPV), negative predictive value (NPV), positive likelihood ratio, negative likelihood ratio, and diagnostic efficacy were calculated.

\section{Results}

Our study included 82 cases with 171 breast lesions. The ages ranged from 29 to 71 years with a mean age of $49.298 \pm 10.75$ (mean $\pm \mathrm{SD}$ ). Upon correlating with the final diagnosis either by histological analysis of postoperative pathology, biopsy samples, fine-needle aspiration cytology (151 lesions were pathologically proved), or close follow up (applied only on 20 lesions that were categorized as Breast Imaging-Reporting and Data System (BIRADS) 2 and showed stationary course for 1 year in a way to confirm their benign nature); $51 / 171$ (29.8\%) lesions were benign, while $120 / 171$ (70.2\%) were malignant. The different pathological entities within benign and malignant groups were seen in Table 1 .

According to CEM findings, lesions were classified into enhancing $(150 / 171,87.7 \%)$ and non-enhancing (21/171) (12.3\%). Non-enhancing lesions were categorized as BIRADS2. Out of 21 non-enhancing lesions, 14/ 21 (81\%) were benign, while $7 / 21(19 \%)$ were malignant. Out of 150 enhancing lesions 113/150 (75\%) were

Table 1 The distribution of different pathological entities

\begin{tabular}{lll}
\hline Diagnosis & Number of lesions & $\%$ \\
\hline Fibroadenoma & $22 / 171$ & 12.9 \\
Inflammatory & $5 / 171$ & 2.9 \\
UDH & $3 / 171$ & 1.8 \\
Focal Adenosis and fibrocystic mastopathy & $15 / 171$ & 8.8 \\
Benign precancerous & $6 / 171$ & 3.5 \\
IDC & $89 / 171$ & 52 \\
ILC & $24 / 171$ & 14 \\
DCIS and Paget & $2 / 171$ & 1.2 \\
DCIS & $5 / 171$ & 2.9 \\
\hline
\end{tabular}

malignant, while 37/150 (25\%) were benign. Enhancing lesions were further classified into enhancing mass lesions 82/150 (54.7\%), enhancing non-mass lesions 59/ 150 (39.3\%), enhancing foci 9/150 (6\%) (Figs. 1 and 2). In reference to the MRI morphology descriptors of benign and malignant breast lesions, 40/171 (23.4\%) lesions were considered benign (assigned BIRADS 2 and 3 categories), while 131/171 (76.6\%) lesions were considered malignant (assigned BIRADS 4 and 5). According to the histopathology results (151 pathologically proved lesions) or close follow up (applied only on 20 lesions that were categorized as BIRADS 2 and showed stationary course for 1 year in a way to confirm their benign nature), 113 lesions were true positives, 18 were false positives, 7 lesions were false negatives, and 33 were true negatives. The 7 false-negative lesions with contrastenhanced spectral mammography (CESM) were a case diagnosed as DCIS and the remaining 6 were additional

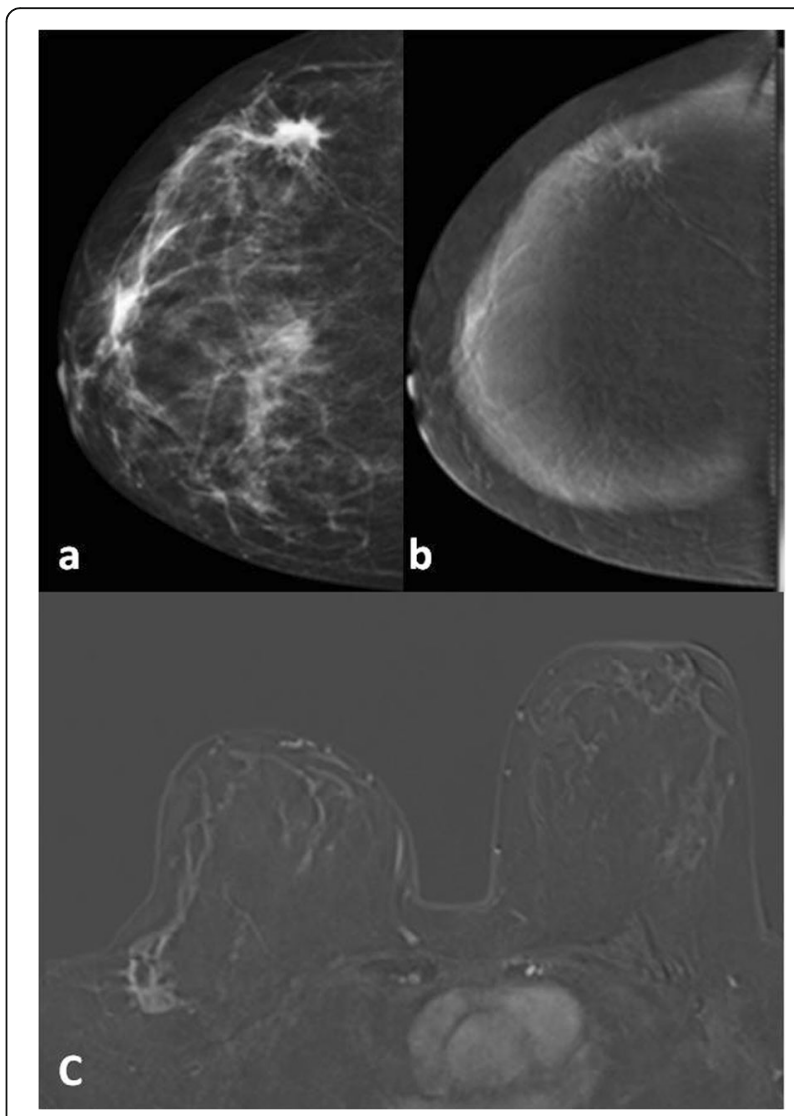

Fig. 1 Forty-six-year-old patient presented by a lump in the right breast. Sonomammography (SM) revealed a speculated mass lesion at right breast upper outer quadrant (UOQ) (a). CEM showed an irregular speculated heterogeneous moderate enhancing mass lesion in right UOQ with associated heterogeneous linear non-mass enhancement (b). DCE-MRI showed an irregular speculated heterogeneous intense enhancing mass lesion in right UOQ with associated linear heterogeneous non-mass enhancement (c). The final diagnosis was invasive lobular carcinoma with LCIS 


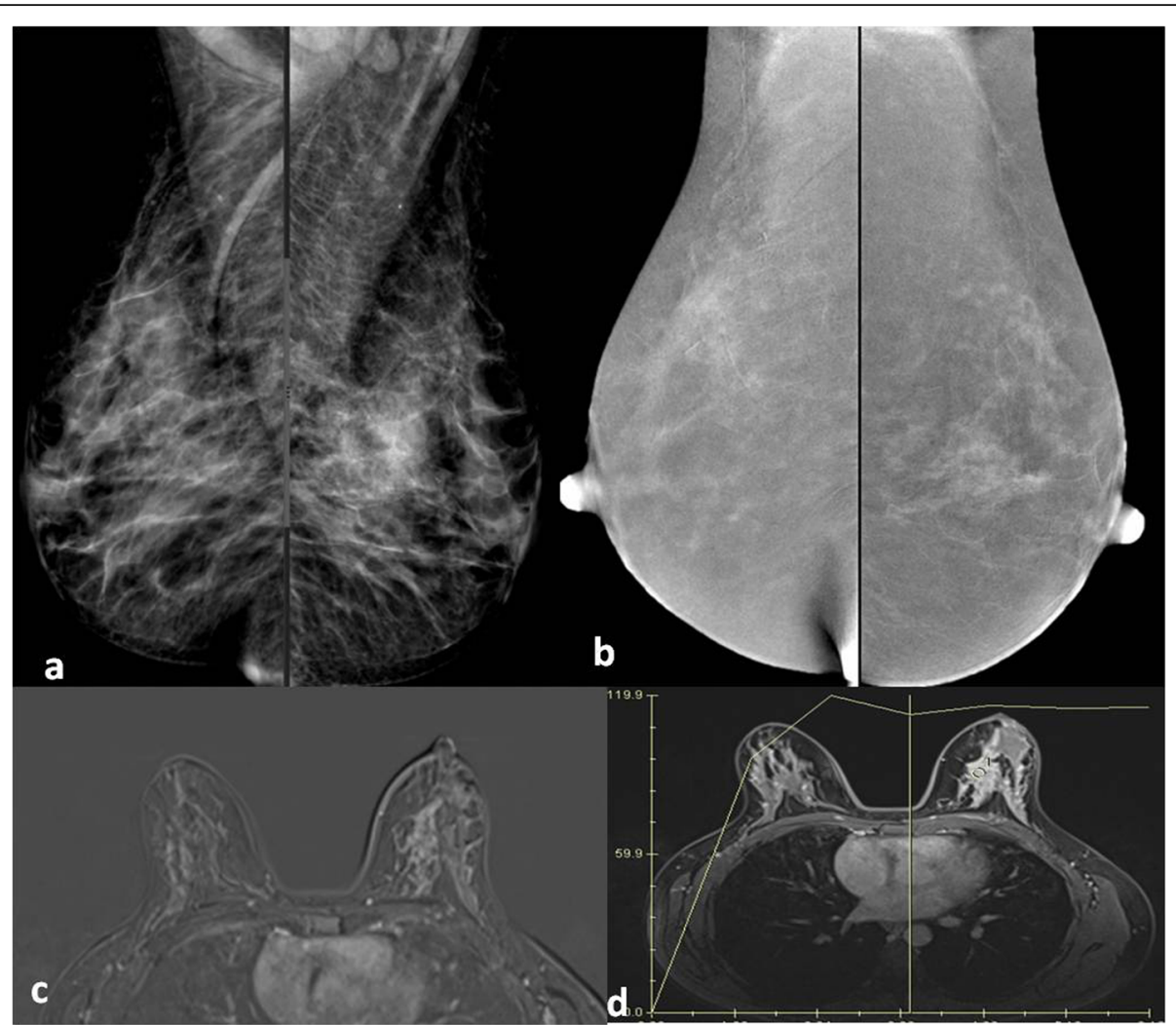

Fig. 2 Thirty-eight-year-old patient came for screening, SM revealed left upper inner quadrant (UIQ) focal asymmetry with overlying concomitant microcalcific clusters (a). CEM showed left UIQ focal asymmetry with overlying concomitant microcalcific clusters (b). MRI showed left breast segmental heterogeneous non-mass enhancement; on plotting time signal intensity curve, it showed type 2 (plateau curve) (c, d). The final diagnosis was ductal carcinoma in situ

malignant lesions identified only on DCE-MRI (Fig. 3). The 18 false-positive (FP) lesions of CESM were 5 inflammatory lesions, 8 atypical fibroadenomas, 4 precancerous lesions (1 sclerosing adenosis, $2 \mathrm{ADH}$, and 1 papillomatosis), and one focal adenosis (Fig. 4).

According to DCE-MRI findings, lesions were classified into enhancing $(158 / 171,92.4 \%)$ and nonenhancing lesions (13/171, 7.6\%). The thirteen nonenhancing lesions were benign. Out of 158 enhancing lesions, 120/158 (76\%) were malignant, while 38/158 (24\%) were benign. Enhancing lesions were classified into enhancing mass lesions (97/158, 61.4\%), enhancing non-mass lesions $(46 / 158,29.1 \%)$, and enhancing foci (15/158 9.5\%) (Figs. 1 and 2). A BIRADS category was given for each lesion; 35/171 (20.5\%) lesions were benign (BIRADS 1, 2, and 3) and 136/171 (79.5\%) lesions were malignant (BIRADS 4 and 5). After revising the pathology, 120 lesions were true positives, 16 were false positives, 0 lesions were false negative, and 35 were true negatives.

The $16 \mathrm{FP}$ lesions of DCE-MRI were 4 inflammatory lesions, 4 atypical fibroadenomas, 3 precancerous lesions, 1 UDH lesion, and 4 focal adenosis (Fig. 5).
The calculated CE-MRI sensitivity, specificity, positive likelihood, negative likelihood, positive predictive value, negative predictive value, and diagnostic accuracy were $100 \%, 68.63 \%, 3.19,0,88.24 \%, 100 \%$, and $90.64 \%$, respectively (Table 2).

The tumor multiplicity was assessed by CEM and MRI in reference to histopathology. CEM detected 22 additional lesions (sensitivity 85\%), while MRI detected 26 additional lesions (sensitivity 100\%)

\section{Discussion}

Full-field digital mammography is accused of having low sensitivity and specificity especially in the dense breast due to overlapping glandular tissue.

Both CE-MRI and CEM have the advantage of providing morphological and functional information as they depend on neovascularity and angiogenesis of lesions [2].

Dynamic contrast-enhanced MRI breast has been used in the assessment of indeterminate mammographic lesions for a long time [11]. The disadvantages of CE-MRI are mainly its relatively high cost, long examination time, limited availability compared to the availability of 

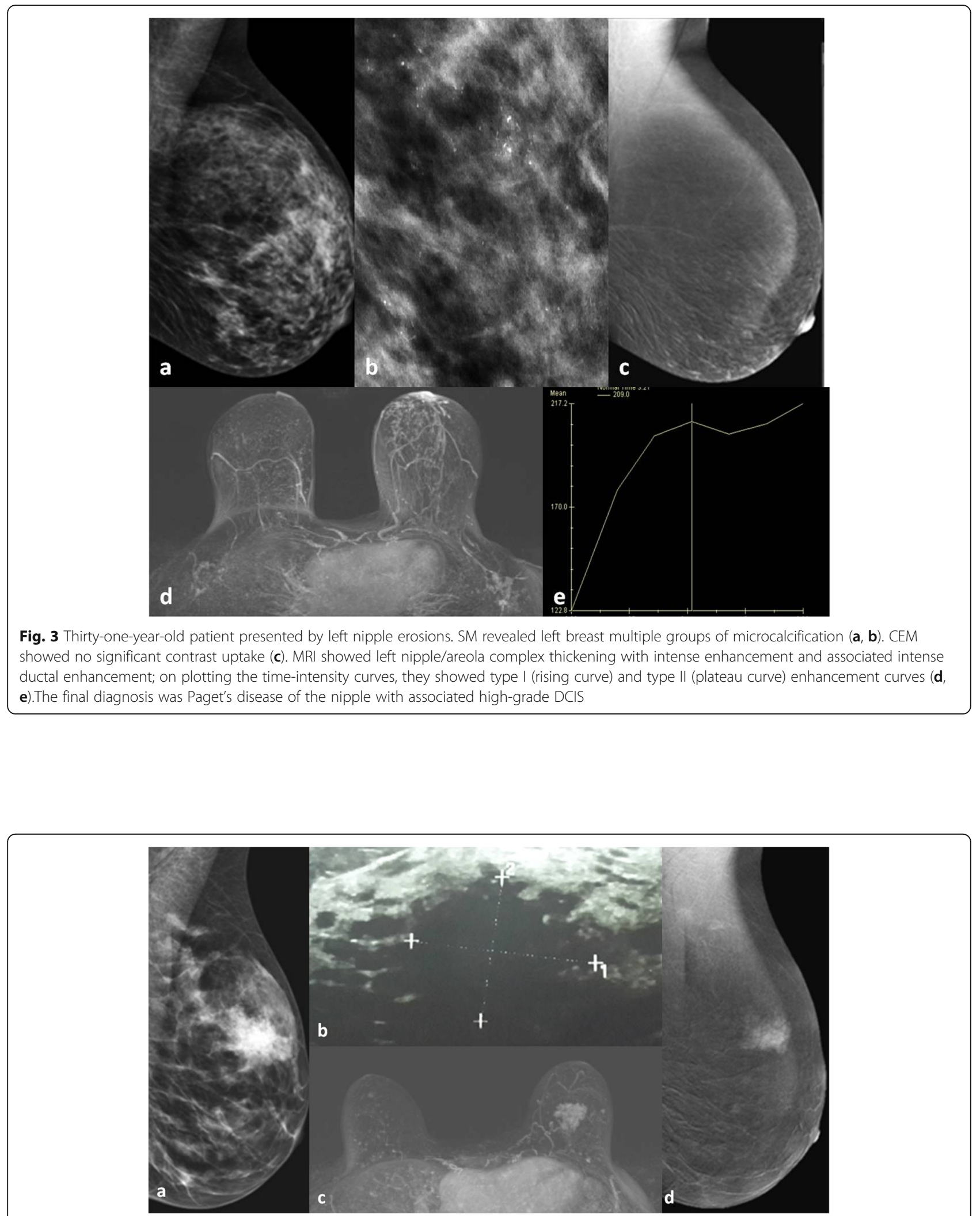

Fig. 4 Thirty-year-old patient with a left breast lump. SM revealed left breast UOQ speculated mass lesions (a, b). MRI showed an irregular not circumscribed speculated intense heterogeneously enhancing mass lesion (c). CEM showed an irregular not circumscribed speculated intensely heterogeneously enhancing mass lesion in left UOQ (d). The final diagnosis was sclerosing adenosis 


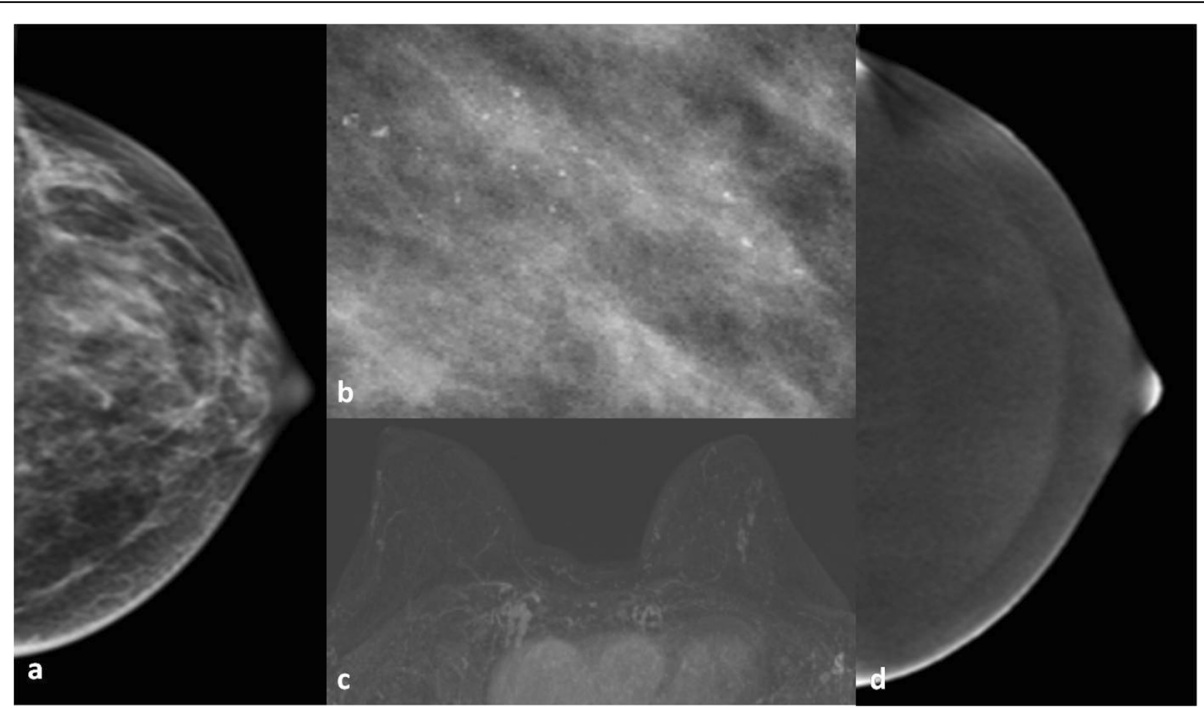

Fig. 5 Sixty-six-year-old patient came for screening with strong positive family history. SM revealed left breast UOQ segmental microcalcifications with no underlying lesions by ultrasound $(\mathbf{a}, \mathbf{b})$. MRI showed left breast linear faint non-mass enhancement (c). CEM showed no significant contrast uptake (d). The final diagnosis was atypical ductal hyperplasia

mammography machine, and non-visualization of calcification [12].

Contrast-enhanced mammography uses a dual-energy technique performed after contrast administration to identify and characterize lesions based on angiogenesis, as well as morphologic features and density [9]. Also, low-energy images of CEM could detect microcalcifications, architectural distortion, and non-enhancing lesions [12].

The main disadvantage in CEM is that it lacks kinematic information about tumor enhancement [13].

Our study revealed $17 / 21$ (81\%) out of the nonenhancing lesions were benign, while $4 / 21$ (19\%) were malignant; out of 150 enhancing lesions, 113/150 (75\%) were malignant, while $37 / 150$ (25\%) were benign. These results are comparable to results of Kamal et al. [14] who found that no enhancement was noted in 66/211 lesions (31.3\%): 60/66 (90.9\%) benign and $6 / 66(9.1 \%)$ malignant lesions, while enhancement was observed in 145/211 lesions (68.7\%):

Table 2 Comparison of CEM and DCE-MRI breast diagnostic indices

\begin{tabular}{llllllll}
\hline & \multicolumn{2}{l}{ CEM } & & \multicolumn{2}{l}{ DCE-MRI } & \multirow{2}{p}{} \\
\cline { 2 - 3 } & Value & $95 \% \mathrm{Cl}$ & & Value & $95 \% \mathrm{Cl}$ & \\
\hline Sensitivity & $94.17 \%$ & 88.35 to 97.62 & $100.00 \%$ & 96.97 to 100.00 & 0.014 \\
Specificity & $64.71 \%$ & 50.07 to 77.57 & $68.63 \%$ & 54.11 to 80.89 & 0.674 \\
PPV & $86.26 \%$ & 81.20 to 90.13 & $88.24 \%$ & 83.33 to 91.84 & 0.628 \\
NPV & $82.50 \%$ & 69.08 to 90.87 & $100.00 \%$ & & 0.013 \\
Accuracy & $85.38 \%$ & 79.18 to 90.31 & & $90.64 \%$ & 85.25 to 94.56 & 0.134 \\
\hline
\end{tabular}

42/145 (29\%) benign and 103/145 (71\%) malignant lesions $(p$ value $\leq 0.001)$ [14].

We found that out of 158 enhancing lesions, 120/158 (76\%) were malignant, while 38/158 (24\%) were benign, and thirteen non-enhancing lesions were benign so our results agree with that of Bennani-Baiti et. al. [15].

Our study revealed that DCE-MRI sensitivity and NPV were slightly yet significantly higher than that of CEM ( $p$ value 0.014 and 0.013 , respectively). The overall accuracy of DCE-MRI was better than that of CESM; however, no statistically significant difference could be detected.

Our results were comparable with Fallenberg et al. that showed that DCE-MRI sensitivity was slightly but significantly superior to CESM ( $p$ value <0.001) [13].

Yousef et al. [16] concluded that CEM and MRI were equal in the sensitivity; however, their study was conducted on twenty cases only [16].

Elfiky et al. [17] and Yasin and El Ghany [12] did a comparative study between CESM and CE MRI; they concluded that contrast-enhanced spectral mammography (CESM) showed slightly lower sensitivity $(88.89 \%, 94.1 \%)$ than BMRI $(96.30 \%$ and $100 \%$ respectively) [12, 17].

However, Łuczyńska et al. [18] found that diagnoses based on CESM are slightly more reliable than those based on breast MRI. The sensitivity of CESM examination was $100 \%$, higher than the $93 \%$ sensitivity of breast MRI ( $p \leq 0.04)$. The accuracy of the CESM exam (79\%) was also higher than that of breast MRI (73\%) in their study, but this difference was not statistically significant. NPV was $100 \%$ for CESM and only $65 \%$ for breast MRI $(p<0.001)[18]$. 
The specificity of CE-MRI was slightly higher than that of CEM in our study but this was not a statistically significant difference. Fallenberg et al. [13] found that the specificity of CESM was better than that of MRI. This could be attributed to the difference in population between their study and our study as their study included only cases with pathologically proven index lesion, yet in our study we included sonomammography indeterminate lesions including both benign and malignant pathologies [13].

In another study done by Xing et al. [19], the sensitivity, PPV, and NPV of CEM were comparable to those of MRI. However, the specificity of CEM was higher than that of MRI [19].

Regarding the assessment of multiplicity in our study in reference to histopathology, CE-MRI was better than CEM in the detection of multiplicity.

Our results were comparable to Jochelson et al. that concluded that CESM had a lower sensitivity for depicting additional ipsilateral cancers than breast MRI [20].

However, Łuczyńska et al. found that CESM detected multifocal breast cancers in all cases studied [18]

\section{Limitation of the study}

The assessment in the study was limited by the absence of a standardized BIRADS lexicon for CEM examination; however, we applied the 2013 MRI BIRADS lexicon morphology descriptors. A standardized lexicon of morphology descriptors seen on CEM would provide the optimal analysis and reporting of enhancing lesions detected in the breast.

\section{Conclusion}

Contrast-enhanced mammography and dynamic contrast-enhanced MRI improved the characterization of breast lesions. CEM showed slightly lower sensitivity and accuracy compared to MRI however because of being relative ease, available, cheap, and acceptable by women; CEM can replace DC-MRI as a problem-solving tool in the characterization of indeterminate breast lesions.

\section{Abbreviations \\ BIRADS: Breast Imaging-Reporting and Data System; CC: Craniocaudal; CEM: Contrast-enhanced mammogram; CESM: Contrast-enhanced spectral mammography; DCE: Dynamic contrast-enhanced; FP: False positive; IDC: Invasive ductal carcinoma; ILC: Invasive lobular carcinoma; MRI: Magnetic resonance imaging; MLO: Mediolateral oblique; NPV: Negative predictive value; PPV: Positive predictive value; SM: Sonomammography; SPSS: Statistical Package for the Social Sciences; UDH: Usual ductal hyperplasia; UOQ: Upper outer quadrant; UIQ: Upper inner quadrant}

\section{Acknowledgements}

Not applicable

\section{Authors' contributions}

RK wrote the manuscript. MH collected patient data and is responsible for correspondence to the journal. MG participated in the design of the study and performed the statistical analysis. SM conceived of the study and participated in its design. MH collected surgical data. All authors have read and approved the manuscript.

Funding

No funding sources.

\section{Availability of data and materials}

The datasets used and analyzed during the current study are available from the corresponding author on reasonable request.

Ethics approval and consent to participate

The study was approved by the ethical committee of the Faculty of Medicine, Cairo University, with ethical approval number 1-15-12-21. An informed written consent was taken from all subjects.

\section{Consent for publication}

All patients included in this research gave written consent to publish the data contained within this study.

\section{Competing interests}

No financial or non-financial competing interests.

\section{Author details}

${ }^{1}$ Department of Diagnostic and Interventional Radiology, Faculty of Medicine, Cairo University, Cairo, Egypt. ${ }^{2}$ Department of Surgical Oncology, National Cancer Institute, Cairo University, Cairo, Egypt. ${ }^{3}$ Department of Diagnostic and Interventional Radiology, National Cancer Institute, Cairo University, Cairo, Egypt.

Received: 10 January 2020 Accepted: 15 April 2020

Published online: 24 April 2020

\section{References}

1. Mostafa AAE, Eltomey MA, Elaggan AM et al (2019) Automated breast ultrasound (ABUS) as a screening tool: initial experience. Egypt J Radiol Nucl Med 50:37

2. Zhu X, Huang J, Zhang K, Xia L et al (2018) Diagnostic value of contrast enhanced spectral mammography for screening breast cancer: systematic review and meta-analysis. Clinical breast cancer 18(5):e985-e995

3. Sadeghi-Naini A, Suraweera H, Tran WT et al (2017) Breast-Lesion Characterization using textural features of quantitative ultrasound parametric maps. Sci Rep 7(1):13638

4. Taşkın F, Polat Y, Erdoğdu IH et al (2018) Problem-solving breast MRI: useful or a source of new problems? Diagn Interv Radiol. Sep 24(5):255-261

5. Mann RM, cho N, Moy L (2019). Breast MRI : sate of the art. Radiology 292(3);520536

6. Lourenco AP, Mainiero MP (2016) Incorporating imaging into the locoregional management of breast cancer. Semin Radiat Oncol 26(1):17-24

7. Bennani-Baiti B, Baltzer PA (2017) MR imaging for diagnosis of malignancy in mammographic microcalcifications: a systematic review and metaanalysis. Radiology 283(3):692-701

8. Patel BK, Lobbes MBI, Lewin J (2018) Contrast enhanced spectral mammography: a review. Semin Ultrasound CT MR 39(1):70-79

9. Perry H, Phillips J, Dialani $V$ et al (2019) Contrast-enhanced mammography: a systematic guide to interpretation and reporting. AJR Am J Roentgenol 212(1):222-231

10. Houben IP, Vanwetswinkel S, Kalia V et al (2019) Contrast-enhanced spectral mammography in the evaluation of breast suspicious calcifications: diagnostic accuracy and impact on surgical management. Acta Radiol 60(9): 1110-1117

11. Yin J, Yang J, Jiang Z (2019) Classification of breast mass lesions on dynamic contrast-enhanced magnetic resonance imaging by a computer-assisted diagnosis system based on quantitative analysis. Oncology Letters 17(3): 2623-2630

12. Yasin R, El Ghany EA (2019) BIRADS 4 breast lesions; comparison of contrastenhanced spectral mammography and contrast enhanced MRI. Egypt J Nucl Med 50:34

13. Fallenberg EM, Schmitzberger FF, Amer $\mathrm{H}$ et al (2017) Contrast-enhanced spectral mammography vs. mammography and MRI - clinical performance in a multi-reader evaluation. Eur Radiol 27(7):2752-2764 
14. Kamal RM, Helal M, Wessam R et al (2015) Contrast-enhanced spectral mammography: Impact of the qualitative morphology descriptors on the diagnosis of breast lesions. Eur Radiol 84(6):P1049-P1055

15. Bennani-Baiti B, Bennani-Baiti N, Baltzer PA (2016) Diagnostic performance of breast magnetic resonance imaging in non-calcified equivocal breast findings: results from a systematic review and meta-analysis. PLoS One. 11(8):e0160346

16. Yousef Ahmed F, Khater Hamada M, Jameel Lara M et al., (2018) Contrastenhanced spectral mammography versus magnetic resonance imaging in the assessment of breast masses. Benha Med J. 35(1). Page: 5-12

17. Elfiky SM, Elsaid NA, Azeb EA et al (2018) Comparison between the role of contrast enhanced mammography and dynamic contrast enhanced MRI in the assessment of breast cancer recurrence. Egypt J Hosp Med 73(1):5875-5885

18. Łuczyńska E, Heinze-Paluchowska S, Hendrick E et al (2015) Comparison between breast MRI and contrast-enhanced spectral mammography. Med Sci Monit 21:1358-1356

19. Xing D, Lv Y, Sun B et al (2019) Diagnostic value of contrast-enhanced spectral mammography in comparison to magnetic resonance imaging in breast lesions. J Comput Assist Tomogr. 43(2):245-251

20. Jochelson MS, Dershaw DD, Sung JS et al (2013) Bilateral contrast-enhanced dual-energy digital mammography: feasibility and comparison with conventional digital mammography and MR imaging in women with known breast carcinoma. Radiology. 266:743-751

\section{Publisher's Note}

Springer Nature remains neutral with regard to jurisdictional claims in published maps and institutional affiliations.

\section{Submit your manuscript to a SpringerOpen ${ }^{\circ}$ journal and benefit from:}

- Convenient online submission

- Rigorous peer review

- Open access: articles freely available online

- High visibility within the field

- Retaining the copyright to your article

Submit your next manuscript at $\boldsymbol{\nabla}$ springeropen.com 